\title{
EMBEDDINGS OF TOPOLOGICAL LATTICE-ORDERED GROUPS
}

\author{
BY \\ ROBERT L. MADELL( $\left.{ }^{1}\right)$
}

1. Introduction. A topological lattice-ordered group (notation: $t l$-group) is a triple $(G, \leqq, T)$ (henceforth denoted simply by $G$ ) where $G$ is a group, $\leqq$ a partial order, and $T$ a topology on the underlying set $|G|$ of $G$ such that (i) $(G, \leqq)$ is a lattice-ordered group (notation: l-group), (ii) $(G, T)$ is a topological group, and (iii) $(|G|, \leqq, T)$ is a topological lattice. It is assumed throughout that topological spaces are $T_{1}$. Familiar examples are $R^{n}$ with the component-wise order and the real valued continuous functions on the unit interval with the point-open topology. With similar results in mind for topological lattices [1], [4], topological semilattices [14, pp. 33-72], and l-groups [13, pp. 88-89] we consider the following questions.

(i) Under what conditions does there exist a one-to-one continuous lattice homomorphism $\pi$ embedding $G$ in the Cartesian product of totally ordered topological lattices $\left\{C_{\alpha} \mid \alpha \in A\right\}$ with the pointwise order on the product? If $G$ is representable, as an l-group, can the $C_{\alpha}$ be taken as totally ordered $t$-groups (notation: to-groups) and $\pi$ as a group homomorphism?

(ii) Assuming that $G$ can be so embedded, under what conditions is $\pi$ : $G \rightarrow G \pi$ even a homeomorphism, where $G \pi$ inherits its topology as a subspace of the full product?

Let $G$ be an $l$-group. The symbol $e$ shall be used throughout to denote the identity of the group under discussion. We use $G^{+}$to designate $\{z \in G \mid z \geqq e\}$ and the elements $x, y \in G$ are called disjoint if $|x| \wedge|y|=e$ where for $z \in G,|z|=z \vee z^{-1}$. A set $C \subseteq G$ is convex in $G$ if from $c_{1}, c_{2} \in C, x \in G, c_{1} \leqq x \leqq c_{2}$ it follows that $x \in C$. An l-subgroup of $G$ is a subgroup which is also a sublattice. An l-ideal of $G$ is a convex normal $l$-subgroup of $G$. If $M$ is a convex $l$-subgroup of $G$ then there is a unique natural way to partially order the right cosets $G / M$ of $M$ so that $G / M$ is a lattice and the natural map $\pi: G \rightarrow G / M$ is a lattice homomorphism $((a \vee b) \pi=a \pi \vee b \pi,(a \wedge b) \pi=a \pi \wedge b \pi)$. If $M$ is an $l$-ideal then $G / M$ is an $l$-group and $\pi$ is an l-homomorphism (a group and lattice homomorphism). If $G$ is a $t$-group and $M$ is a closed convex $l$-subgroup then there is a unique natural way to topologize $G / M$ so that $G / M$ is a topological lattice and the natural map $\pi: G \rightarrow G / M$ is continuous and open. If $M$ is a closed $l$-ideal then $G / M$ is a $t /$-group and

Received by the editors August 13, 1968 and, in revised form, July 2, 1969.

${ }^{1}$ ) This work was supported in part by National Science Foundation grant GP-7083 and represents a portion of the author's doctoral dissertation written under the direction of Professor Charles Holland. 
$\pi: G \rightarrow G / M$ provides a one-to-one correspondence between the closed convex l-subgroups of $G$ which contain $M$ and the closed convex $l$-subgroups of $G / M$. If $G$ and $H$ are $t$-groups and $\gamma: G \rightarrow H$ is a continuous $l$-homomorphism the kernel of $\gamma$ is a closed $l$-ideal of $G$.

If $\left\{G_{\alpha} \mid \alpha \in A\right\}$ are l-groups, the direct product $\prod_{\alpha \in A} G_{\alpha}$ is the set of all functions $f$ on $A$ such that the $\alpha$ th component $f(\alpha)$ is in $G_{\alpha}$; the operations in $\prod_{\alpha \in A} G_{\alpha}$ are performed component-wise. Then $\prod_{\alpha \in A} G_{\alpha}$ is an l-group. An l-group $G$ is said to be a subdirect sum of $\left\{G_{\alpha} \mid \alpha \in A\right\}$ if $G$ is an l-subgroup of $\prod_{\alpha \in A} G_{\alpha}$ and each projection map $\pi_{\alpha}: G \rightarrow G_{\alpha}$ is a surjection. If each $G_{\alpha}$ is a $t$ l-group, $\prod_{\alpha \in A} G_{\alpha}$ shall be considered to have the Cartesian topology so $\prod_{\alpha \in A} G_{\alpha}$ is also a $t$-group. A $t$-group $G$ is said to be a $t$-subdirect sum of $t$-groups $\left\{G_{\alpha} \mid \alpha \in A\right\}$ if $G$ is a subdirect sum of $\left\{G_{\alpha} \mid \alpha \in A\right\}$ as an l-group and the topology on $G$ is that inherited from $\prod_{\alpha \in A} G_{\alpha}$. An l-group $G$ is said to be representable if there is an $l$-isomorphism (one-to-one l-homomorphism) of $G$ onto a subdirect sum of totally ordered l-groups (notation: o-groups). If $\left\{C_{\alpha} \mid \alpha \in A\right\}$ are topological lattices, $\prod_{\alpha \in A} C_{\alpha}$ shall denote the set of all functions $f$ on $A$ such that the $\alpha$ th component $f(\alpha)$ is in $C_{\alpha}$; the lattice operations are performed pointwise and $\prod_{\alpha \in A} C_{\alpha}$ is given the Cartesian topology so it is a topological lattice. If $G$ and $G^{*}$ are $o$-groups and $\pi: G \rightarrow G^{*}$ is a group isomorphism satisfying $a \leqq b$ if and only if $a \pi \leqq b \pi$ then $\pi$ is an $o$-isomorphism.

Since every $t$-group $G$ is a topological group its underlying space is homogeneous. Furthermore, if $M$ is a subgroup of $G, M^{c}$ (the closure of $M$ ) is also a subgroup of $G$. Since $G$ is an $l$-group, for each $g \in G, g \neq e$, every convex $l$-subgroup $M_{g}$ of $G$ maximal with respect to missing $g$ is prime (if $a \wedge b \in M_{g}, a \in M_{g}$ or $b \in M_{g}$ ) so $G / M_{g}$ is totally ordered. Every prime convex $l$-subgroup of $G$ contains a minimal prime convex $l$-subgroup which is an $l$-ideal if $G$ is representable [8, Theorem 3.1]. Since $G$ is a $T_{1}$ topological lattice, $L(g)=\{x \in G \mid x \leqq g\}=\{x \in G \mid x \vee g=g\}$ and $U(g)=\{x \in G \mid x \geqq g\}=\{x \in G \mid x \wedge g=g\}$ are closed.

Let $G$ be a $t /$-group and $M$ a closed convex $l$-subgroup of $G$. For $g \in G$ let $g^{*}: G / M \rightarrow G / M$ denote the homeomorphism defined by $(M x) g^{*}=M x g$ and let $g^{+}=g \vee e$. If $A$ and $B$ are subsets of $G$ let $A \backslash B=\{x \in G \mid x \in A, x \notin B\}$. Let $A^{\prime}=G \backslash A$. We use $\varnothing$ to denote the empty set. For background information in l-groups and topological groups see [7], [13], [17]. The author is grateful to the referee especially for his simplifications of the original proofs of Proposition 7 and Lemma 11.

2. Examples. Every l-group $G$ may be endowed with the discrete topology and therefore be considered to be a $t$-group. Furthermore, if $G$ is totally ordered, $G$ with the interval topology is a to-group. On the other hand, if $G$ is a totally ordered topological lattice and $g_{1}, g_{2} \in G$ with $g_{1}<g_{2}$ then $\left\{g \mid g_{1}<g<g_{2}\right\}$ is open. There do exist to-groups whose topologies lie properly between the interval and discrete topologies [15, p. 55] although even for totally ordered topological lattices this cannot happen in the connected locally compact case. 
THEOREM 1. Let $G$ be a connected nonzero to-group. Then $G$ is o-isomorphic to the o-group $\boldsymbol{R}$ of real numbers under addition in their natural order. If $G$ is also locally compact then its topology is precisely the interval topology.

Proof. Since at least the open intervals are open in $G$ every nonzero convex subgroup of $G$ is open and so closed and hence equal to all of $G$. Hence $G$ is $o$ isomorphic to a subgroup of $\boldsymbol{R}$. By connectivity, $G$ is $o$-isomorphic to all of $\boldsymbol{R}$. It is shown in [3, Theorem 4] that a connected totally ordered topological lattice has the interval topology if and only if it is locally compact.

Using the main results of this paper we shall show (Theorem 15) that the first of the examples below is the only connected, locally compact, $n$-dimensional tl-group.

(i) Real Euclidean $n$-space $\boldsymbol{R}^{n}$ as the usual topological group and with $\left(x_{1}, x_{2}, \ldots, x_{n}\right) \geqq\left(y_{1}, y_{2}, \ldots, y_{n}\right)$ if and only if $x_{i} \geqq y_{i}, i=1,2, \ldots, n$.

(ii) The group of real valued continuous functions on the unit interval with the point open topology and $f \leqq g$ if and only if for each real number $x$ in the unit interval $f(x) \leqq g(x)$.

(iii) Every $t$-subdirect sum of $t$-groups.

(iv) Every completely distributive l-group with its $\alpha$-topology, [16].

(v) In [18] it is shown that for any l-group $G$, any filter basis $\mathscr{F}$ of $l$-ideals of $G$ with $\bigcap_{F \in F} F=\{e\}$ is a neighborhood basis of $e$ for a unique topology with which $G$ is a $t$ l-group.

THEOREM 2. No nonzero tl-group $G$ is compact.

Proof. The collection $\{L(g) \mid g \in G\}$ has the finite intersection property so if $G$ were compact there would exist $x \in \bigcap_{g \in G} L(g)$ contradicting the fact that an $l$-group can have no smallest element.

THEOREM 3. If $G$ is a tl-group and $g \in G$ then the homotopy groups $\Pi_{n}(G, g)$, $n \geqq 1$, are trivial.

Proof. It suffices by homogeneity to show that $\Pi_{n}(G, e)$ is trivial. We show that $\Pi_{1}(G, e)$ is trivial and remark that the proof is easily generalized.

Let $I$ denote the unit interval and let $f$ be a continuous map from $I$ into $G$ with $f(0)=f(1)=e$. Let $f^{+}(t)=f(t) \vee e$ and let $f^{-}(t)=f(t) \wedge e$ and define $F^{+}: I \times I \rightarrow G$ and $F^{-}: I \times I \rightarrow G$ by $F^{+}(s, t)=f^{+}(t) \wedge f^{+}(t-t s)$ and $F^{-}(s, t)=f^{-}(t) \vee f^{-}(t-t s)$ respectively. It is then easy to check that if $H(s, t)=F^{+}(s, t) F^{-}(s, t)$ then $H(0, t)=f(t), H(1, t)=e$, and $H(s, 0)=H(s, 1)=e$.

3. The $T_{1}$ radical. The theory developed in this section is closely analogous to portions of the theory of the distributive radical developed in [9].

Proposition 4. Let $M$ be a convex l-subgroup of a tl-group $G$. Then $M^{c}$ is a convex l-subgroup. If $M$ is an l-ideal then so is $M^{c}$. 
Proof. We only need to show the convexity of $M^{c}$. First observe that $\left(M^{c}\right)^{+}=$ $\left(M^{+}\right)^{c}$. For if $x \in\left(M^{c}\right)^{+}$and $U$ is a neighborhood of $x$ we may pick a neighborhood $V$ of $x$ with $V \vee e \subseteq U$. Then if $m \in V \cap M, m \vee e \in U \cap M^{+}$so $x \in\left(M^{+}\right)^{c}$. The other inclusion is clear. Now let $a \leqq z \leqq b$ with $a, b \in M^{c}$. Then $e \leqq z a^{-1} \leqq b a^{-1}$ so

$$
z a^{-1}=z a^{-1} \wedge b a^{-1} \in z a^{-1} \wedge\left(M^{c}\right)^{+}=z a^{-1} \wedge\left(M^{+}\right)^{c} \subseteq\left(z a^{-1} \wedge M^{+}\right)^{c} \subseteq M^{c}
$$

so $z=\left(z a^{-1}\right) a \in M^{c}$.

If we now define the $T_{1}$ radical, $T_{1}(G)$, to be the intersection of the closures of the minimal prime convex $l$-subgroups of the $t$-group $G$ the following results are immediate.

THEOREM 5. Let $G$ be a tl-group and let $\left\{M_{\alpha} \mid \alpha \in A\right\}$ denote the minimal prime convex l-subgroups of $G$. Then the natural map $\pi: G \rightarrow \prod_{\alpha \in A}\left(G / M_{\alpha}^{c}\right)$ is a continuous lattice homomorphism. It is one-to-one if and only if $T_{1}(G)=\{e\}$.

THEOREM 6. Let $G$ be a representable tl-group. Then $G$ is l-isomorphic to a t-subdirect sum of to-groups by an l-isomorphism which is continuous if and only if $T_{1}(G)=\{e\}$.

In order to show that $T_{1}(G)$ has the usual radical properties we need the following result which is an analogue of a result in [9, Lemma 3.3].

Proposition 7. Let $G$ be a tl-group and $M$ a prime convex l-subgroup of $G$. Then if $M$ contains a closed prime convex l-subgroup $N$ of $G, M$ is open and hence also closed.

Proof. Assume $M \neq N$ and let $\pi: G \rightarrow G / N$ be the natural map. Then $M \pi$ is convex, clearly without least or greatest element, and hence open. Since $\pi$ is continuous and $M=(M \pi) \pi^{-1}, M$ is open and so also closed in $G$.

THEOREM 8. Let $G$ be a tl-group. Then the following hold.

(i) $T_{1}(G)$ is a closed l-ideal of $G$.

(ii) If $M$ is a convex l-subgroup of $G$ then $M \cap T_{1}(G)=T_{1}(M)$.

(iii) $T_{1}\left(T_{1}(G)\right)=T_{1}(G)$.

(iv) $T_{1}\left(G / T_{1}(G)\right)=\{e\}$.

(v) $T_{1}(G)=\{e\}$ if and only if $T_{1}(C(g))=\{e\}$ for all $g \in G$ where $C(g)$ denotes the convex l-subgroup of $G$ generated by $g$.

Proof. (i) We only need normality. But for $x \in G$,

$$
x^{-1} T_{1}(G) x=x^{-1}\left(\bigcap_{\alpha \in A} M_{\alpha}^{c}\right) x \subseteq \bigcap_{\alpha \in A} x^{-1} M_{\alpha}^{c} x=\bigcap_{\alpha \in A}\left(x^{-1} M_{\alpha} x\right)^{c}=T_{1}(G) .
$$

(ii) Certainly $T_{1}(M) \subseteq M \cap T_{1}(G)$ since if $C$ is a closed prime convex l-subgroup of $G$ then $C \cap M$ is a closed prime convex l-subgroup of $M$. Now let $g \in M \cap T_{1}(G)$, $g>e$, and suppose by way of contradiction that $K$ is a closed prime convex $l$-subgroup of $M$ with $g \notin K$. Let $M_{1}$ be a maximal convex $l$-subgroup of $M$ with respect to missing $g$ such that $K \subseteq M_{1}$. By Proposition $7, M_{1}$ is closed in $M$. 
Let $N$ be a maximal convex $l$-subgroup of $G$ with respect to missing $g$ and such that $M_{1} \subseteq N$. Then $N \cap M=M_{1}$ and $N$ is prime in $G$. We claim that $g \notin N^{c}$ for the desired contradiction. For suppose $g \in N^{c}$. Then $g=g \wedge g \in g \wedge N^{c} \subseteq(g \wedge N)^{c}$ so $g=e \vee g \in e \vee(g \wedge N)^{c} \subseteq(e \vee(g \wedge N))^{c}$. But $e \vee(g \wedge N) \subseteq N \cap M=M_{1}$ so $g \in M_{1}^{c}$ $\cap M=M_{1}$ since $M_{1}$ is closed in $M$. This contradicts the choice of $M_{1}$.

(iii) This follows immediately from (ii).

(iv) If $M_{\alpha}^{c}$ is the closure of a minimal prime convex l-subgroup of $G$ then $M_{\alpha}^{c} / T_{1}(G)$ is a closed prime convex l-subgroup of $G / T_{1}(G)$. Since $\bigcap_{\alpha \in A} M_{\alpha}^{c}=T_{1}(G)$, the result is clear.

(v) If $T_{1}(G)=\{e\}$ then (ii) certainly implies that $T_{1}(C(g))=\{e\}$, for all $g \in G$. Suppose on the other hand that $T_{1}(C(g))=\{e\}$ for all $g \in G$ and suppose $x \in T_{1}(G)$. Then $x \in T_{1}(G) \cap C(x)=T_{1}(C(x))$ so $x=e$.

In [9] a convex l-subgroup $M$ of an l-group $G$ is called closed if whenever $\left\{g_{\alpha} \mid \alpha \in A\right\} \subseteq M$ and $\bigvee_{\alpha \in A} g_{\alpha}$ exists then $\bigvee_{\alpha \in A} g_{\alpha} \in M$. To avoid confusion with topological closure we shall say that $M$ is $L$-closed.

THEOREM 9. Let $G$ be a tl-group and $M$ a convex l-subgroup of $G$. Then if $M$ is $L$-closed, $M$ is topologically closed.

Proof. It suffices to show that for $g \in M^{c}$ with $g \geqq e$ that $g \in M$. But $g=$ $(g \vee e) \wedge g \in\left(M^{c} \vee e\right) \wedge g \subseteq[(M \vee e) \wedge g]^{c}$. Let $K=[(M \vee e) \wedge g]=\{x \in M \mid e \leqq x \leqq g\}$. Then $g=\bigvee_{k \in K} k$. For if $K \leqq h$ but $g \leqq h$ then the open set $[L(h)]^{\prime}$ satisfies $g \in[L(h)]^{\prime}$ but $[L(h)]^{\prime} \cap K=\varnothing$ for a contradiction. Since $M$ is $L$-closed, $g \in M$.

Since the distributive radical $D(G)$ of [9] was defined to be the intersection of the $L$-closures of the minimal prime convex $l$-subgroups of $G$, we have $T_{1}(G) \subseteq D(G)$. In particular, we have the following.

CoROllaRY 10. If $G$ is a completely distributive tl-group then $T_{1}(G)=\{e\}$.

Proof. It is shown in [9, Theorem 3.10] that an l-group $G$ is completely distributive if and only if $D(G)=\{e\}$.

It should be observed that if $G$ is the $t$-group of example (ii) then $T_{1}(G)=\{e\}$ although it can be shown that $D(G)=G$ [12]. In general then, $T_{1}(G) \varsubsetneqq D(G)$. Furthermore, the l-group $H$ of continuous real valued functions on the unit interval may be topologized so that with that topology it is a $t$-group satisfying $T_{1}(H)=H$. (By [18], one such topology is obtained by taking as a basis about $e$ the collection of sets of the form $N_{\varepsilon}=\{f \mid m\{x:|f(x)| \geqq \varepsilon\}<\varepsilon\}$ where $m$ denotes Lebesgue measure, $\varepsilon>0$.) Thus, by simultaneously looking at example (ii) it is seen that the $T_{1}$ radical cannot in general be described l-group-theoretically although this can be done for a certain class of $t l$-groups [15, Corollary 9.8].

4. Homeomorphic embeddings. Let $G$ be a $t$-group with $T_{1}(G)=\{e\}$. Then there exists a collection $\left\{N_{\alpha} \mid \alpha \in A\right\}$ of closed prime convex $l$-subgroups of $G$ with $\bigcap_{\alpha \in A} N_{\alpha}=\{e\}$ and so the natural map $\pi: G \rightarrow \prod_{\alpha \in A}\left(G / N_{\alpha}\right)$ is a one-to-one 
continuous lattice homomorphism. It is natural to ask under what conditions $\pi: G \rightarrow G \pi\left(G \pi\right.$ with the topology inherited from $\left.\prod_{\kappa \in A}\left(G / N_{\alpha}\right)\right)$ is a homeomorphism.

LEMMA 11. Let $G$ be a tl-group and let $B$ and $N$ be convex l-subgroups of $G$ with $N$ prime and $B \nsubseteq N$. Then $\bar{\pi}: B \rightarrow G / N$ is open where $\bar{\pi}$ is the restriction to $B$ of the natural map $\pi: G \rightarrow G / N$ and $B$ inherits its topology from $G$.

Proof. It will suffice to show that for each neighborhood $W$ of $e$ there exists a neighborhood $U$ of $e$ with $U \pi \subseteq(W \cap B) \bar{\pi}$. For this take $p \in B \backslash N$ and a neighborhood $V$ of $e$ such that $(V \vee V) \wedge|p| \subseteq W$ and $(V \wedge V) \vee|p|^{-1} \subseteq W$. Let $U=V \cap$ $\left\{\left.g \in G|N| p\right|^{-1}<N g<N|p|\right\}$ so $U$ is clearly a neighborhood of $e$. Pick $u \in U$ and suppose $N u \geqq N$. Since $N u=N((u \vee e) \wedge|p|)$ and $(u \vee e) \wedge|p| \in W \cap B$, $u \pi \in(W \cap B) \bar{\pi}$ as desired. The case for $N u \leqq N$ is dual.

THEOREM 12. Let $G$ be a tl-group and let $\left\{N_{\alpha} \mid \alpha \in A\right\}$ be a collection of closed prime convex l-subgroups of $G$ which meet in the identity. Then the natural map $\pi: G \rightarrow \prod_{\alpha \in A}\left(G / N_{\alpha}\right)$ is a homeomorphism from $G$ onto $G \pi$, where $G \pi$ inherits its topology from $\prod_{\alpha \in A}\left(G / N_{\alpha}\right)$, if and only if

$\left.{ }^{*}\right)$ given any neighborhood $O$ of e there exists a finite subset $\alpha(1), \alpha(2), \ldots, \alpha(k)$ of $A$ such that $\bigcap_{i=1}^{k} N_{\alpha(i)} \subseteq O$.

Proof. If $\pi$ is a homeomorphism it is easy to see that $\left(^{*}\right)$ holds. Now suppose that $\left({ }^{*}\right)$ is satisfied. It will suffice to show that $\pi^{-1}$ is continuous at $e \pi$. So let $O$ be a neighborhood of $e$ in $G$. Let $O^{*}$ be a neighborhood of $e$ such that $O^{*} O^{*} \subseteq O$. Using $\left(^{*}\right)$ we can pich $\left\{N_{1}, \ldots, N_{k}\right\} \subseteq\left\{N_{\alpha} \mid \alpha \in A\right\}$ and a neighborhood $O_{1}$ of $e$ with $\left(\bigcap_{i=1}^{k} N_{i}\right) O_{1} \subseteq O^{*}$. We may assume that for each $i, 1 \leqq i \leqq k, B_{i}=\bigcap_{j \neq i} N_{j} \nsubseteq N_{i}$. Pick a neighborhood $V$ of $e$ such that if $V_{i}=V$ for $i=1,2, \ldots, k$ then $\bigvee_{i=1}^{k} V_{i} \subseteq O_{1}$ and $\bigwedge_{i=1}^{k} V_{i} \subseteq O_{1}$. Pick a neighborhood $V^{*}$ of $e$ such that $V^{*} \vee e \subseteq V$ and $V^{*} \wedge e \subseteq V$.

Now let $U=\left\{g \pi \mid N_{i} g \in\left(B_{i} \cap V^{*}\right) \bar{\pi}_{i}, 1 \leqq i \leqq k\right\}$. By Lemma $11,\left(B_{i} \cap V^{*}\right) \bar{\pi}_{i}$ is open in $G / N_{i}$ for $i=1,2, \ldots, k$ so $U$ is a neighborhood of $e \pi$ in $G \pi$. We will show that $U \pi^{-1} \subseteq O$. For pick $g \in U \pi^{-1}$ so $g \pi \in U$. Then $N_{i} g \in\left(B_{i} \cap V^{*}\right) \bar{\pi}_{i}$ so $N_{i}(g \vee e)$ $=N_{i} g \vee N_{i} e=N_{i} b_{i} \vee N_{i} e=N_{i}\left(b_{i} \vee e\right)$ where $b_{i} \in B_{i} \cap V^{*}, \quad i=1,2, \ldots, k$. Then $N_{i}(g \vee e) \in\left(B_{i} \cap V\right) \bar{\pi}_{i}$ so we can let $N_{i}(g \vee e)=N_{i} g_{i}$ with $g_{i} \in B_{i} \cap V$ and $N_{i} g_{i} \geqq N_{i}$ in $G / N_{i}, i=1,2, \ldots, k$. Then for $i=1,2, \ldots, k$,

$$
N_{i}\left(\bigvee_{j=1}^{k} g_{j}\right)=\left(\left(\bigvee_{j=1}^{k} g_{j}\right) \pi\right)(i)=\left(\bigvee_{j=1}^{k}\left(g_{j} \pi\right)\right)(i)=N_{i} g_{i}=N_{i}(g \vee e) .
$$

That is to say, $(g \vee e)\left(\bigvee_{j=1}^{k} g_{j}\right)^{-1} \in \bigcap_{i=1}^{k} N_{i}$. But since $\bigvee_{j=1}^{k} g_{j} \in O_{1}$,

$$
g \vee e=(g \vee e)\left(\bigvee_{j=1}^{k} g_{j}\right)^{-1}\left(\bigvee_{i=1}^{k} g_{j}\right) \in\left(\bigcap_{i=1}^{k} N_{i}\right) O_{1} \subseteq O^{*}
$$

A similar argument will show that $g \wedge e \in O^{*}$ so that $g=(g \vee e)(g \wedge e) \in O^{*} O^{*} \subseteq O$. 
5. Applications. The condition $\left({ }^{*}\right)$ of Theorem 12 is obviously satisfied in the $t /$-group $G$ by any finite collection of closed prime convex $l$-subgroups which meet in the identity of $G$. This section contains some consequences of that fact.

Proposition 13. Let $G$ be a tl-group and $\left\{N_{1}, \ldots, N_{k}\right\}$ a set of prime convex l-subgroups which meet in the identity and which have the property that $\bigcap_{j \neq i} N_{j} \nsubseteq N_{i}$, $i=1,2, \ldots, k$. Then for $i=1,2, \ldots, k, N_{i}$ is closed. Furthermore if $G$ is connected then each $N_{i}$ is an l-ideal and the natural map $\pi: G \rightarrow \prod_{i=1}^{k}\left(G / N_{i}\right)$ is a surjection.

Proof. For $i=1,2, \ldots, k$ pick $a_{i}>e, a_{i} \in\left(\bigcap_{j \neq i} N_{j}\right) \backslash N_{i}$. Then

$$
N_{i}=a_{i}^{\perp}=\left\{z \in G|| z \mid \wedge a_{i}=e\right\} \text {. }
$$

For let $z>e$. If $z \in N_{i}$ then $z \wedge a_{i} \in \bigcap_{j=1}^{k} N_{j}=\{e\}$ so $z \in a_{i}^{\perp}$. On the other hand, if $z \in a_{i}^{+}$then $z \wedge a_{i}=e$ so since $N_{i}$ is prime, $z \in N_{i}$. It follows from the continuity of $\wedge$ that $N_{i}$ is closed, $i=1,2, \ldots, k$.

Let us assume for the moment that $\bar{\pi}_{i}$, the restriction of the natural map $\pi_{i}: G \rightarrow G / N_{i}$ to $B_{i}=\bigcap_{j \neq i} N_{j}$, is a surjection. Then for $x \in G, x N_{i}=b_{i} N_{i}=N_{i} b_{i}=N_{i} x$ for some $b_{i} \in B_{i}$ since $\left|b_{i}\right| \wedge\left|n_{i}\right|=e$ for all $n_{i} \in N_{i}$ and disjoint elements commute. Hence, $N_{i}$ is normal in $G$. Furthermore, for each positive $\left(N_{1} \cdot x_{1}, N_{2}, x_{2}, \ldots, N_{k}-x_{k}\right)$ in $\prod_{i=1}^{k}\left(G / N_{i}\right)$ we have $\left(N_{1} x_{1}, N_{2}, x_{2}, \ldots, N_{k} \cdot x_{k}\right)=\left(N_{1} b_{1}, N_{2} b_{2}, \ldots, N_{k} b_{k}\right)$ $=\left(b_{1} \vee b_{2} \vee \cdots \vee b_{k}\right) \pi$ with $b_{i} \in B_{i}$. Hence, $\pi$ is a surjection.

To show that $\bar{\pi}_{i}$ is a surjection observe that by Lemma $11, B_{i} \bar{\pi}_{i}$ is open in $G / N_{i}$. Now let $N_{i} g \notin B_{i} \bar{\pi}_{i}$. Then $\left(B_{i} \bar{\pi}_{i}\right) g^{*}$ is a neighborhood of $N_{i} g$ and it is easy to check that $\left(B_{i} \bar{\pi}_{i}\right) g^{*} \cap B_{i} \bar{\pi}_{i}=\varnothing$ so $B_{i} \bar{\pi}_{i}$ is also closed in $G / N_{i}$. By connectivity, $B_{i} \bar{\pi}_{i}$ is all of $G / N_{i}$.

COROLlaRY 14. Let $G$ be a connected locally compact tl-group which contains a finite collection of prime concex l-subgroups which meet in the identity. Then $G$ is isomorphic as a $\mathrm{tl}$-group to $\boldsymbol{R}^{k}$ for some integer $k$.

Proof. Every finite collection of subgroups which meets in the identity contains a subcollection $N_{1}, \ldots, N_{k}$ which meets in the identity and also has the property that $\bigcap_{j \neq i} N_{j} \nsubseteq N_{i}, i=1,2, \ldots, k$. Each $G / N_{i}$ is a connected locally compact $t$ group so is isomorphic as a $t /$-group to $R$. Since $\left\{N_{1}, \ldots, N_{k}\right\}$ satisfies $\left(^{*}\right)$ and $\pi$ is a surjection we are done.

Definition. If $L$ is a lattice, then the breadth of $L$, denoted by $\operatorname{Br}(L)$, is the smallest integer $n$ such that any finite subset $F$ of $L$ has a subset $F^{*}$ of at most $n$ elements such that $\bigwedge_{f \in F} f=\bigwedge_{f \bullet \in F^{*}} f^{*}$.

Let $X$ be a locally compact Hausdorff space. We shall use $\operatorname{cov}(X)$ to denote the Lebesgue covering dimension of $X$ and ind $(X)$ to denote the inductive dimension of Urysohn and Menger. Let cd $(X)$ denote the Cohen codimension of $X[10]$.

THEOREM 15. Let $G$ be a connected locally compact tl-group. Then if either $\operatorname{Br}(G)=n, \operatorname{cd}(G)=n, \operatorname{cov}(G)=n$, or ind $(G)=n, G$ is isomorphic as a tl-group to $\boldsymbol{R}^{n}$. 
Proof. Suppose $\operatorname{Br}(G)=n$. Then $G$ does not contain $n+1$ disjoint elements. For if $\left\{a_{1}, \ldots, a_{n}, a_{n+1}\right\}$ is a disjoint set of positive elements and $F=$ $\left\{\bigvee_{i \neq j} a_{i} \mid j=1, \ldots, n, n+1\right\}$, it is easy to check that $\bigwedge_{f \in F} f=e$ but for every proper subset $F^{*}$ of $F, \bigwedge_{r^{*} \in F^{*}} f^{*} \neq e$. Hence there exists an integer $k \leqq n$ such that $G$ has $k$ disjoint elements but not $k+1$. Let $\left\{b_{1}, b_{2}, \ldots, b_{k}\right\}$ be a set of $k$ disjoint elements in $G$. Then each $b_{i}^{+}, i=1, \ldots, k$ is a prime convex $l$-subgroup and $\bigcap_{i=1}^{k} b_{i}^{\perp}=\{e\}$. Furthermore, $b_{j} \in\left(\bigcap_{i \neq j} b_{i}^{\perp}\right) \mid b_{j}^{\perp}$. Then $G$ is isomorphic as a $t$-group to $R^{k}$ so since $\operatorname{Br}(G)=n, k=n$.

Now suppose $\mathrm{cd}(G)=n$. By results of [2], [5], $\mathrm{Br}(G) \leqq n$. Hence, $G$ is isomorphic as a $t$-group to $\boldsymbol{R}^{k}$ for some $k \leqq n$. But $\mathrm{cd}\left(\boldsymbol{R}^{k}\right)=k$ so $k=n$.

Finally, if either $\operatorname{cov}(G)=n$ or ind $(G)=n$ the result follows from the fact that $\operatorname{cd}(G) \leqq n$ since $c d(G) \leqq \operatorname{cov}(G)$ and $c d(G) \leqq$ ind $(G)$ [10, Theorems 5.7, 7.2].

COROLlaRY 16 ([6, TheOREM 9]). The only tl-groups which are at the same time (connected) Lie groups are the tl-groups $\boldsymbol{R}^{n}, n=1,2, \ldots$

\section{REFERENCES}

1. L. W. Anderson, Topological lattices and n-cells, Duke Math. J. 25 (1958), 205-208.

2. - On the breadth and co-dimension of a topological lattice, Pacific J. Math. 9 (1959), 327-333.

3. - On the distributivity and simple connectivity of plane topological lattices, Trans. Amer. Math. Soc. 91 (1959), 102-112.

4. - The existence of continuous lattice homomorphisms, J. London Math. Soc. 37 (1962), 60-62.

5. L. W. Anderson and L. E. Ward, Jr., A structure theorem for topological lattices, Proc. Glasgow Math. Assoc. 5 (1961), 1-3.

6. G. Birkhoff, Lattice-ordered Lie groups, Comment. Math. Helv., Speiser Festschrift (1945), 209-217.

7. - Lattice theory, 3rd ed., Amer. Math. Soc. Colloq. Publ., Vol. 25, Providence, R. I., 1967.

8. R. D. Byrd, Complete distributivity in lattice-ordered groups, Pacific J. Math. 20 (1967), 423-432.

9. R. D. Byrd and J. T. Lloyd, Closed subgroups and complete distributivity in lattice-ordered groups, Math. Z. 101 (1967), 123-130.

10. H. Cohen, A cohomological definition of dimension for locally compact Hausdorff spaces, Duke Math. J. 21 (1954), 209-224.

11. P. Conrad, The structure of a lattice-ordered group with a finite number of disjoint elements, Michigan Math. J. 7 (1960), 171-180.

12. J. Ellis, Group topological convergence in completely distributive lattice ordered groups, Doctoral dissertation, Tulane Univ., New Orleans, La., 1968.

13. L. Fuchs, Partially ordered algebraic systems, Pergamon Press (Addison-Wesley), New York, 1963.

14. J. D. Lawson, Vietoris mappings and embeddings of topological semilattices, Doctoral dissertation, The Univ. of Tennessee, Knoxville, 1967.

15. R. L. Madell, Topological lattice ordered groups, Doctoral dissertation, The Univ. of Wisconsin, Madison, 1968. 
16. R. L. Madell, On complete distributivity and $\alpha$-convergence (to appear).

17. L. Pontriagin, Topological groups, Princeton Univ. Press, Princeton, N. J., 1939.

18. B. Smarda, Topologies in l-groups, Arch. Math. 3 (1967), 69-81.

UNIVERSITY OF TENNESSEE,

KNOXVILle, TeNNESSEE

UNIVERSITY OF WISCONSIN,

Madison, Wisconsin 